\title{
USO DE ANTIMICROBIANOS SISTÉMICOS POR ODONTÓLOGOS GENERALES PARA EL TRATAMIENTO DE PATOLOGÍAS PULPARES Y PERIAPICALES DE ORIGEN PULPAR
}

\author{
${ }^{1}$ Álix Forero Orejarena, ${ }^{1}$ Marcela Arregocés Solano, ${ }^{1}$ Juan Carlos Ávila Vera, \\ ${ }^{1}$ Elaine Ortega Herrera, ${ }^{1}$ Diana Paola Ortiz Bueno, ${ }^{2}$ Guillermo Gómez Moya. \\ ${ }^{1}$ Estudiante de la Especialización en Endodoncia, U. Santo Tomás. ${ }^{2}$ Médico y Cirujano, Docente U. Santo Tomás, \\ Profesor Asociado U. Industrial de Santander.
}

Autor responsable de correspondencia: Álix Forero Orejarena

Correo electrónico: alixodoonto@hotmail.com

\section{RESUMEN}

Objetivo: Describir los hábitos de uso de antimicrobianos sistémicos por parte de odontólogos generales en el manejo de las patologías pulpares y periapicales de origen pulpar.

Materiales y métodos: Estudio observacional descriptivo de corte transversal, con población de estudio conformada por 150 odontólogos generales, residentes en la ciudad de Bucaramanga, a quienes mediante entrevista directa, se aplicó un cuestionario previamente validado. Se obtuvo información de diferentes variables de interés relacionadas con el uso de antimicrobianos sistémicos en las patologías referidas. Se aplicaron pruebas de estadística descriptiva y de asociación entre variables, $\left(\mathrm{Chi}^{2}, t\right.$ Student, con $\left.\mathrm{p}=0,05\right)$.

Resultados: Entre el $28 \%$ y el $68.6 \%$ de los odontólogos generales usaron siempre algún antimicrobiano para patologías pulpares, así éstos no estuviesen indicados. Entre el $22.7 \%$ y el $72 \%$ de los odontólogos, prescribieron algún antimicrobiano de tipo profiláctico en pacientes con compromiso sistémico. La amoxicilina fue el antimicrobiano más prescrito en todos los casos. Los esquemas de administración de antimicrobianos atendieron las recomendaciones establecidas para los casos tratados.

Conclusión: Los antimicrobianos sistémicos son ampliamente utilizados por odontólogos generales en las patologías pulpares y periapicales de origen pulpar, incluidos los casos en que no están indicados. [Forero A, Arregoces M, Ávila JC, Ortega E, Ortiz DP, Gómez G. Uso de antimicrobianos sistémicos por odontólogos generales para el manejo de patologías pulpares y periapicales de origen pulpar. Ustasalud 2009; 8: $75-82]$

Palabras clave: Antimicrobiano, patología pulpar, patología periapical, endodoncia

\section{SYSTEMIC ANTIMICROBIAL USE BY GENERAL DENTISTS FOR THE TREATMENT OF PULP AND PERIAPICAL PATHOLOGIES OF PULPAL ORIGIN}

\begin{abstract}
Objective: To describe the habits of systemic antimicrobial use by general dentists in the treatment of pulp and periapical pathology of pulpal origins.

Methods: An observational descriptive cross-sectional study was done; the surveyed population consisted of 150 general dentists living at Bucaramanga city who, through a direct interview, answered a previously validated questionnaire. Information was gathered from different variables of interest related to the use of systemic antimicrobials in the pathologies in question. Descriptive statistics and tests of association between variables $\left(\mathrm{Chi}^{2}, \mathrm{t}\right.$ Student $\left.\mathrm{p}=0.05\right)$ were developed based on the information recollected.

Results: Between $28 \%$ and $68.6 \%$ of the dentists always use some antimicrobial for pulp pathologies without being indicated. Between $22.7 \%$ and $72 \%$ of dentists use some type of prophylactic antimicrobial in patients with a systemic condition. Amoxicillin was the most commonly used antibiotic in all cases. Administration schemes of antimicrobials attended the established recommendations for the treated cases.

Conclusion: A high proportion of general dentists in Bucaramanga use systemic antimicrobials in the treatment of pulp and periapical pathology of pulpal origins even if not indicated.
\end{abstract}

Key words: Antimicrobial, pulpal pathology, periapical pathology, endodontics.

Recibido para publicación: 8 de septiembre de 2009. Aceptado para publicación: 2 de febrero de 2010 . 


\section{INTRODUCCIÓN}

Las infecciones odontogénicas de origen pulpar y periapical son muy frecuentes en las consultas odontológicas y para su tratamiento, por lo general, no se indica el uso de antimicrobianos sistémicos, excepto cuando hay presencia de periodontitis apical aguda supurativa o una condición clínica sistémica en el paciente. ${ }^{1}$ Sin embargo, en diversas ocasiones, los odontólogos usan antimicrobianos para el tratamiento de todas las patologías pulpares. ${ }^{2}$ Existen publicaciones que refieren la sobremedicación antimicrobiana, como por ejemplo, donde el $46 \%$ de los odontólogos prescriben antimicrobianos en tratamientos endodónticos y en retratamientos ${ }^{2}$. Se ha reportado que el 53.93\% de los endodoncistas prescribe antimicrobianos para tratar periodontitis apical aguda sin inflamación, el $16.76 \%$ los prescribe para pulpitis irreversible y el $12 \%$ para periodontitis apical crónica. ${ }^{3}$

En otro estudio, se observó que el $62.5 \%$ de los pacientes se automedicaron con antimicrobianos y el $32.5 \%$ eran prescritos por los odontólogos. ${ }^{4}$ También, se ha encontrado que el $21.68 \%$ de los odontólogos, prescriben antimicrobianos en periodontitis apical crónica no supurativa y el $20.69 \%$ en periodontitis apical crónica supurativa. ${ }^{5}$

Por otra parte, la resistencia bacteriana ha aumentado en los últimos años por el uso inadecuado de antimicrobianos por vía sistémica, unida al lento avance en la creación de nuevos medicamentos anti infecciosos y la búsqueda de estrategias que permitan su control. En Colombia, las tasas de resistencia a antimicrobianos encontradas son superiores a las mostradas por estudios de vigilancia en Estados Unidos, Europa y otros países de América Latina. ${ }^{6}$ Los porcentajes de resistencia bacteriana oscilaron, para estos estudios entre un $61 \%$ y $83 \%{ }^{6}$

Los antimicrobianos con fines sistémicos en las patologías pulpares se pueden utilizar con fines profilácticos o curativos y se indican cuando hay certeza de un proceso infeccioso, cuando se considera que haya respuesta al antimicrobiano y cuando sea una infección grave. ${ }^{7,8}$ Los antimicrobianos se deben administrar para tratar infecciones con posibilidad de diseminación sistémica y cuando los hallazgos clínicos no indican una posible resolución espontánea. Los signos y síntomas que sugieren el compromiso sistémico son la fiebre, el edema y la inflamación, como por ejemplo en la periodontitis apical aguda supurativa, que es la única patología de origen pulpar y periapical que requiere medicación antimicrobiana. ${ }^{8,9}$ En pacientes con compromiso sistémico se indican en forma profiláctica para todas las patologías pulpares.
Las patologías pulpares, que originan una lesión endodóntica periapical, producen salida de sustancias irritantes hacia los tejidos perirradiculares, los que sufren diferente grado de efecto a su exposición y presentan diversos cambios tisulares. Las patologías periapicales de origen pulpar se clasificaron como periodontitis apical aguda no supurativa, periodontitis apical aguda supurativa, periodontitis apical crónica no supurativa y periodontitis apical crónica supurativa. ${ }^{10}$ Las patologías pulpares se clasificaron como pulpitis irreversible aguda y pulpitis irreversible crónica. ${ }^{11}$

El propósito de este estudio fue describir el uso de antimicrobianos sistémicos por parte de odontólogos generales, en el tratamiento de las patologías pulpares y periapicales de origen pulpar.

\section{MATERIALES Y MÉTODOS}

Se realizó un estudio observacional descriptivo de corte transversal, con una población de estudio conformada por 150 odontólogos generales, residentes en la ciudad de Bucaramanga. El tamaño de la muestra fue calculado de acuerdo a la prevalencia del uso de antimicrobianos en patologías pulpares y periapicales de origen pulpar, encontrada en la literatura revisada, que varió entre el $12 \%$ y el $53.9 \%,{ }^{3}$ con un nivel de confianza de $95 \%$, un poder de $80 \%$ y un nivel de significancia del 5\% (Programa Epiinfo versión 6.04).

Previo al inicio del estudio, se realizó una prueba piloto, con la participación de 15 odontólogos generales, seleccionados de manera aleatoria, que cumplían los requisitos de elegibilidad establecidos para este estudio, odontólogos generales hombres o mujeres graduados que ejercieran en la ciudad de Bucaramanga, de cualquier edad, y que expresaran voluntariamente su participación en el estudio. Esta prueba, permitió validar el cuestionario aplicado en la entrevista personal, estandarizar procedimientos dentro del grupo de trabajo, como también identificar y resolver aspectos de tipo logístico.

Los odontólogos generales participantes en el estudio se seleccionaron de manera aleatoria, mediante un listado conformado a partir del registro disponible en la Secretaría de Salud Departamental, de la información existente en el directorio telefónico de la ciudad y de un listado suministrado por algunas de las casas comerciales que proveen productos odontológicos.

A los odontólogos generales seleccionados se les contactó telefónicamente y se les invitó a participar en el estudio, quienes expresaron su aceptación en forma voluntaria. Se obtuvo el consentimiento informado oral de cada uno de los participantes y 
posteriormente, se aplicó el cuestionario mediante entrevista personal.

La variable dependiente más importante fue el uso de antimicrobianos sistémicos por parte de los odontólogos generales en patologías pulpares y periapicales de origen pulpar. Otras variables fueron, el tipo de antimicrobiano prescrito, la dosificación empleada, el intervalo de administración, la frecuencia de uso y la frecuencia de solicitud de un antimicrobiano por parte de los pacientes.

Las variables independientes tenidas en cuenta, relacionadas con la población de estudio, fueron el género, la edad, el tiempo de ejercicio profesional, la asistencia a capacitación en el área odontológica clínica, el tiempo promedio de duración de la consulta odontológica.

Además, se tuvieron en cuenta variables relacionadas con la ausencia o presencia de compromiso sistémico en los pacientes atendidos, incluidas endocarditis bacteriana, diabetes mellitus, hemofilia, fiebre reumática y uso de anticoagulantes orales. Se indagó sobre si el propósito de uso de los antimicrobianos tenía fines profilácticos o curativos.

Los aspectos éticos se acogieron a la Resolución 008430 del Ministerio de Salud, al tener en cuenta los principios relacionados con el respeto a la dignidad, la protección de los derechos y la privacidad de los sujetos. El protocolo de investigación recibió concepto favorable del Comité de Investigaciones de la Facultad de Odontología de la Universidad Santo Tomás.
Para el procesamiento de la información, se realizó un análisis estadístico descriptivo univariado, se consideraron las variables de acuerdo con su naturaleza, nivel de clasificación y escalas de medición. Se aplicaron medidas de tendencia central y medidas de dispersión. Se llevó a cabo un análisis bivariado para determinar la relación de las variables independientes de interés con el uso de antimicrobianos sistémicos en patologías pulpares y periapicales, y se aplicaron pruebas de significancia estadística t de Student, $\mathrm{Chi}^{2}$, $\mathrm{p}=0.05$.

\section{RESULTADOS}

Entre los odontólogos generales participantes, hubo predominio del género femenino con $69.3 \%$ (104). El promedio de edad de los participantes fue $36.4 \pm 7.6$ años, con rango entre 24 y 66 años. El promedio del tiempo de ejercicio profesional fue $11.9 \pm 7.4$ años. Del total de encuestados, el 78.7\% (118) había recibido capacitación en áreas clínicas de la odontología diferentes a la especialidad en endodoncia, durante el último año. El tiempo promedio de duración de la consulta odontológica fue $31.7 \pm 17$ minutos.

En relación con el ejercicio profesional, predominó la práctica privada ubicada en su gran mayoría en estrato socioeconómico 4 (42.7\%) (Tabla 1). El uso de antimicrobianos sistémicos por los odontólogos generales, en las patologías pulpares y periapicales de origen pulpar se describe en la Tabla 2.

Tabla 1. Distribución de los odontólogos generales por estrato socioeconómico del sitio de trabajo, Bucaramanga, 2009

\begin{tabular}{cccc}
\hline ESTRATO SOCIOECONÓMICO & NÚMERO & PORCENTAJE (\%) \\
\hline 1 & 2 & 7 & 1.33 \\
2 & 32 & 4.7 \\
3 & 64 & 21.3 \\
4 & 27 & 42.7 & 18.0 \\
& 5 & 18 & 12.0 \\
\hline
\end{tabular}

Tabla 2. Uso de antimicrobianos en patologías pulpares y periapicales por odontólogos generales, Bucaramanga, 2009

\begin{tabular}{lc}
\hline PATOLOGÍA & USO ANTIMICROBIANO \\
\hline Periodontitis apical aguda supurativa
\end{tabular}


El uso de antimicrobianos en las patologías pulpares y periapicales con fines curativos, es aceptable sólo para casos de periodontitis apical aguda supurativa y los resultados indican que los odontólogos generales los prescribieron en $91.3 \%$.

La frecuencia de uso de antimicrobianos sistémicos por los odontólogos generales, en las patologías pulpares y periapicales de origen pulpar se describe en la Tabla 3. En la que sólo el $68.6 \%$ de los odontólogos entrevistados siempre prescribía antimicrobianos en caso de periodontitis apical aguda supurativa. Es frecuente el uso de antimicrobianos sistémicos no indicados en otros casos de este tipo de patologías. El tipo de antimicrobiano sistémico prescrito y su frecuencia de uso se expresan en la Tabla 4.

La amoxicilina fue el antimicrobiano de mayor uso en todas las patologías pulpares y periapicales, con una frecuencia de $78.1 \%$ de los casos.

El uso de los antimicrobianos en las patologías pulpares y periapicales, incluido el tipo de tratamiento (profiláctico o curativo), en aquellos pacientes con compromiso sistémico, se describe en la Tabla No. 5.

Tabla 3. Frecuencia de uso antimicrobiano en patología pulpar y periapical por odontólogos generales, Bucaramanga 2009

\begin{tabular}{|c|c|c|c|}
\hline \multirow[b]{2}{*}{ PATOLOGÍA } & \multicolumn{3}{|c|}{ FRECUENCIA } \\
\hline & SIEMPRE & $\begin{array}{l}\text { LA MAYORÍA DE } \\
\text { LAS VECES }\end{array}$ & OCASIONAL \\
\hline Periodontitis apical aguda supurativa & $68.6 \%$ & $24.1 \%$ & $7.3 \%$ \\
\hline Periodontitis apical crónica supurativa & $64.0 \%$ & $22.0 \%$ & $14.0 \%$ \\
\hline Periodontitis apical aguda no supurativa & $68.5 \%$ & $16.4 \%$ & $15.1 \%$ \\
\hline Periodontitis apical crónica no supurativa & $64.0 \%$ & $22.0 \%$ & $14.0 \%$ \\
\hline Necrosis pulpar & $51.4 \%$ & $23.6 \%$ & $25.0 \%$ \\
\hline Pulpitis irreversible aguda & $41.8 \%$ & $25.4 \%$ & $32.7 \%$ \\
\hline Pulpitis irreversible crónica & $28.0 \%$ & $36.0 \%$ & $36.0 \%$ \\
\hline
\end{tabular}

Tabla 4. Antimicrobianos de mayor uso en patologías pulpares y periodontales por odontólogos generales, Bucaramanga 2009

\begin{tabular}{lcc} 
PATOLOGÍA & ANTIMICROBIANO & $\begin{array}{c}\text { FRECUENCIA USO } \\
\mathbf{n}(\%)\end{array}$ \\
\hline \multirow{2}{*}{ Pulpitis irreversible aguda } & Amoxicilina & $43(78.1)$ \\
& Cefalexina & $4(7.3)$ \\
Periodontitis apical crónica no supurativa & Amoxicilina & $30(68.0)$ \\
Necrosis pulpar & Dicloxacilina & $4(8.0)$ \\
& Amoxacilina & $54(67.0)$ \\
Periodontitis apical crónica supurativa & Dicloxacilina & $8(11.1)$ \\
& Amoxacilina & $73(67.0)$ \\
Pulpitis irreversible crónica & Dicloxacilina & $14(12.8)$ \\
Periodontitis apical aguda no supurativa & Amoxicilina & $32(54.0)$ \\
& Azitromicina & $7(14.0)$ \\
Periodontitis apical aguda supurativa & Amoxacilina & $42(57.5)$ \\
& Dicloxacilina & $11(15.1)$ \\
& Amoxacilina & $74(54.0)$ \\
\hline
\end{tabular}


La frecuencia de uso de antimicrobianos con fines profilácticos, en patologías pulpares y periapicales para pacientes con compromiso sistémico es baja, la frecuencia es de uso aceptable sólo en casos de endocarditis bacteriana (72\%). En cualquier patología pulpar y periapical para pacientes con compromiso sistémico el uso de antimicrobianos se debe realizar con fines profilácticos.

La asociación del uso de antimicrobianos sistémicos en patologías pulpares y periapicales y su relación con variables independientes de interés, se describe en la Tabla 6.

Tabla 5. Frecuencia de uso antimicrobiano en pacientes con compromiso sistémico por odontólogos generales, Bucaramanga 2009

\begin{tabular}{|c|c|c|c|c|c|c|}
\hline \multirow[b]{2}{*}{ PATOLOGÍA } & \multicolumn{2}{|c|}{ USO DE ANTIMICROBIANOS } & \multicolumn{4}{|c|}{ PROPÓSITO DEL TRATAMIENTO } \\
\hline & $\begin{array}{c}\text { SI } \\
\text { N (\%) }\end{array}$ & $\begin{array}{c}\text { NO } \\
\text { N (\%) }\end{array}$ & $\begin{array}{c}\text { PROFILÁCTICO } \\
\text { N (\%) }\end{array}$ & $\begin{array}{c}\text { CURATIVO } \\
\text { N (\%) }\end{array}$ & $\begin{array}{c}\text { PROFILÁCTICO Y } \\
\text { CURATIVO } \\
\text { N (\%) }\end{array}$ & $\begin{array}{c}\text { NINGUNO } \\
\text { N (\%) }\end{array}$ \\
\hline Endocarditis bacteriana & $141(94.0)$ & $9(6.0)$ & $108(72.0)$ & $1(0.7)$ & $33(22.0)$ & $8(5.3)$ \\
\hline Diabetes mellitus & $106(70.7)$ & $44(29.3)$ & $69(46.0)$ & $7(4.7)$ & $35(23.3)$ & $39(26.0)$ \\
\hline Hemofilia & $46(30.7)$ & $104(69.3)$ & $33(22.0)$ & $4(2.7)$ & $17(11.3)$ & $96(64.0)$ \\
\hline Anticoagulación & $52(34.7)$ & $98(65.3)$ & $34(22.7)$ & $4(2.7)$ & $20(13.3)$ & $92(61.3)$ \\
\hline Fiebre reumática & 89 (59.3) & $61(40.7)$ & $67(44.7)$ & $4(2.7)$ & $20(13.3)$ & $59(39.3)$ \\
\hline
\end{tabular}

Tabla 6. Uso de antimicrobianos en patologías pulpares y periapicales según variables independientes, Bucaramanga, 2009.

\begin{tabular}{|c|c|c|c|c|}
\hline \multirow[b]{2}{*}{ PATOLOGÍA } & \multirow[b]{2}{*}{ VARIABLES INDEPENDIENTES } & \multicolumn{2}{|c|}{ USO ANTIMICROBIANOS } & \multirow[b]{2}{*}{ VALOR P* } \\
\hline & & $\begin{array}{c}\text { SI } \\
\mathrm{n}(\%)\end{array}$ & $\begin{array}{c}\text { NO } \\
\mathrm{n}(\%)\end{array}$ & \\
\hline \multirow{3}{*}{ Pulpitis irreversible aguda } & Estrato socio-económico & & & \\
\hline & 1,2 y 3 & $45(81.8)$ & $64(67.4 \%)$ & \\
\hline & 4,5 y 6 & $10(18.2)$ & $31(32.6 \%)$ & $0.056^{*}$ \\
\hline \multirow{6}{*}{ Pulpitis irreversible crónica } & Género & & & \multirow{3}{*}{$0.033^{*}$} \\
\hline & Femenino & $29(58)$ & $75(75)$ & \\
\hline & Masculino & $21(42)$ & $25(25)$ & \\
\hline & Trabaja inst. particulares & & & \multirow{3}{*}{$0.015^{*}$} \\
\hline & $\mathrm{Si}$ & $32(64)$ & $43(43)$ & \\
\hline & No & $18(36)$ & $57(57)$ & \\
\hline \multirow{6}{*}{$\begin{array}{l}\text { Periodontitis apical aguda } \\
\text { supurativa }\end{array}$} & Edad & & & \multirow{3}{*}{$0.019^{*}$} \\
\hline & 35 años o más & 78 (56.9) & $3(23.1)$ & \\
\hline & Menos de 35 años & $59(43.1)$ & $10(84.6)$ & \\
\hline & Años de egresado & & & \multirow{3}{*}{$0.008^{* *}$} \\
\hline & 11 o más años & $74(54)$ & $2(15.4)$ & \\
\hline & Menos de 11 años & $63(46)$ & $11(84.6)$ & \\
\hline \multirow{6}{*}{$\begin{array}{l}\text { Periodontitis apical aguda no } \\
\text { supurativa }\end{array}$} & Trabaja en inst. particular & & & \multirow{3}{*}{$0.034^{*}$} \\
\hline & $\mathrm{Si}$ & 43 (58.9) & $32(41.6)$ & \\
\hline & No & $30(41.1)$ & $45(58.4)$ & \\
\hline & Duración de la consulta & & & \multirow{3}{*}{$0.032^{* *}$} \\
\hline & Mayor de 20 minutos & $28(38.4)$ & $43(55.8)$ & \\
\hline & 20 minutos o menos & $45(61.6)$ & $34(44.2)$ & \\
\hline
\end{tabular}

${ }^{*} \mathrm{Chi}^{2}{ }^{* *} t$ de Student 
Para la pulpitis irreversible aguda no se hallaron diferencias estadísticamente significativas según el estrato socioeconómico, es destacado que el $81 \%$ de los odontólogos que usaron antimicrobianos para esta patología, trabaja la mayor parte de su tiempo en instituciones que se encuentran en estratos 1,2 y $3(p=0.056)$.

En la pulpitis irreversible aguda, la mayoría de odontólogos que usan antimicrobianos, son del género femenino (58\%) lo cual evidencia menor porcentaje de no uso por parte de los hombres (42\%) $(p=0.033)$. De igual manera, se encontró diferencia estadística para uso de antimicrobianos, en relación con el hecho de trabajar en instituciones particulares, incluida la consulta privada $(\mathrm{p}=0.015)$.

Para la pulpitis irreversible crónica se detectaron diferencias significativas cuando se tuvo en cuenta el sitio donde el odontólogo labora $(p=0.015)$. Con respecto a la periodontitis apical aguda supurativa, el uso de antimicrobianos se demostró asociado con la edad de los participantes $(\mathrm{p}=0.019)$ y con el tiempo que lleva de egresado como profesional de odontología $(\mathrm{p}=0.008)$

En cuanto a la periodontitis apical aguda no supurativa, se encontraron diferencias estadísticas relacionadas con el ejercicio profesional en instituciones particulares $(p=0.034)$, ya que el total de profesionales que usan antimicrobianos en esta patología, labora en este tipo de instituciones (58.9\%). De manera similar, se encontraron resultados estadísticos significativos para el uso de antimicrobianos sistémicos, según el tiempo de duración de la consulta $(p=0.032)$, pues el $61.6 \%$ de las consultas odontológicas tenían una duración menor de 20 minutos.

\section{DISCUSIÓN}

Este estudio muestra una frecuencia de uso de antimicrobianos en la pulpitis irreversible aguda de un $36.7 \%$, mientras un estudio en Estados Unidos, estableció prescripción de estos medicamentos de $16.7 \%$ para la misma patología. ${ }^{3} \mathrm{El}$ resultado del uso de antimicrobianos en pulpitis irreversible aguda por odontólogos generales en este estudio, fue mayor comparado con otro estudio en el que se encontró un $5.42 \%$ con una muestra de 1200 odontólogos generales encuestados. ${ }^{5}$

El presente estudio mostró un $72.7 \%$ de uso de antimicrobianos para la periodontitis apical crónica supurativa, mientras que para la periodontitis apical crónica no supurativa fue de $33.3 \%$. Estos resultados son comparativamente mayores a un estudio realizado en Argentina, en el que se prescribían antimicrobianos para la periodontitis api- cal crónica supurativa en un $20.6 \%$ y para la periodontitis apical crónica no supurativa en un $21.6 \% .^{5}$

Para la periodontitis apical aguda no supurativa, se encontró un $48.7 \%$ de uso de antimicrobianos, mientras estudios realizados por el Comité Americano de Endodoncia, ${ }^{5,12}$ y por Whitten y colaboradores encontraron un $33 \%$ y un $67 \%$, respectivamente, de prescripción para esta alteración periapical. ${ }^{13}$ También, se observó un $48 \%$ de uso de antimicrobianos en caso de necrosis pulpar, ésta es una proporción similar a la hallada por Yingling y colaboradores (53.9\%) y mayor a la reportada en otros estudios. ${ }^{3,5,12}$

Este estudio mostró que se usan antimicrobianos para la periodontitis apical aguda supurativa en un $91.3 \%$, similar a otros estudios con un $99.2 \%$ y $87.6 \%{ }^{3,13}$ Los odontólogos generales que ejercen en instituciones particulares tienden a usar más antimicrobianos sistémicos y en los estratos socioeconómicos 1, 2 y 3, posiblemente para prevenir alguna complicación después de la atención al paciente.

Es esencial tener en cuenta que no hay indicación para el uso de antimicrobianos sistémicos en caso de patologías pulpares y periapicales de origen pulpar con fines terapéuticos o profilácticos, excepto en la periodontitis apical aguda supurativa en pacientes con compromiso sistémico. Está demostrado, mediante diversos estudios que su uso no disminuye el dolor pulpar, ni la sensibilidad a la percusión, sin tratar el diente endodónticamente. ${ }^{15,16}$

En este estudio, se encontró que el antimicrobiano más usado en la periodontitis apical aguda supurativa fue la amoxicilina en un 54\%, esta proporción es mayor a la encontrada en un estudio en Cuba, de $29.9 \% .^{14}$

Según los estudios revisados, en el tratamiento de las pulpitis irreversibles agudas y crónicas no hay indicación para el uso de antimicrobianos, sólo se elimina el tejido pulpar y posteriormente, se realiza el tratamiento del conducto radicular. ${ }^{3,5,17,18}$ Otros estudios demostraban que en la necrosis pulpar no hay indicación para el uso de antimicrobianos y el tratamiento debe ser la terapia endodóntica. ${ }^{3,5}$

En el tratamiento de la periodontitis apical crónica supurativa no se ha incluido el uso de antimicrobianos para controlar la infección causada por microorganismos; sólo se indica la terapia endodóntica como tal, con sólo realizar la apertura del conducto se rompe la anaerobiosis. ${ }^{8}$ Estudios demuestran que esta patología es manejada por 
los odontólogos generales con antimicrobianos mediante uso indiscriminado en cuanto a elección, dosis y frecuencia de administración. ${ }^{19}$

El uso de antimicrobianos sólo está indicado en caso de periodontitis apical aguda supurativa, en la cual existe un edema difuso, que puede ir desde la zona adyacente al diente afectado hasta invadir los espacios aponeuróticos con la posibilidad de comprometer la vida del paciente. Al principio del proceso es difícil localizar el diente afectado, presenta cierta movilidad y está ligeramente extruido. ${ }^{20}$ Algunos estudios reportan que dentro de los antimicrobianos de elección en esta patología, está la penicilina $\mathrm{V}$ que es la de elección, luego la amoxicilina de 500 mg y la amoxicilina más el ácido clavulónico, ya que conserva su actividad contra las beta-lactamasas producidas por organismos que se asocian a infecciones odontogénicas. ${ }^{8,18,21}$ La duración ideal de la terapia antimicrobiana debe ser de 3 a 7 días. En caso del paciente alérgico a las penicilinas se usa la clindamicina. ${ }^{8,21}$

Este estudio muestra que el antimicrobiano que más usan los odontólogos generales encuestados para las patologías pulpares y periapicales de origen pulpar y periapical fue la amoxicilina con dosis de $500 \mathrm{mg}$, de 6 a 7 días con un intervalo de 6 a 8 horas.

Similar a otros estudios revisados, en los que se encontró que el esquema terapéutico de los antimicrobianos sistémicos. en caso de periodontitis apical aguda supurativa es la penicilina 1.000.000 U.I. una ampolla cada 12 horas por dos días, amoxicilina $500 \mathrm{mg}$ vía oral una cada 8 horas por 5 a 7 días; clindamicina $300 \mathrm{mg}$ vía oral una cada 8 horas por 5 a 7 días, en caso de personas alérgicas a las penicilinas. ${ }^{8,21}$

Estudios revisados muestran que para el tratamiento de todas las patologías pulpares y periapicales de origen pulpar en pacientes con compromiso sistémico, como endocarditis bacteriana, diabetes mellitus, hemofilia, pacientes con medicación anticoagulante y fiebre reumática, se usan siempre antimicrobianos sistémicos con fines profilácticos, la amoxicilina es la primera elección..$^{21,22}$ En otros estudios, se observaron los esquemas profilácticos de elección, entre ellos la amoxicilina 2.0 gr vía oral, una hora antes del procedimiento; en pacientes alérgicos, clindamicina, $600 \mathrm{mg}$ vía oral una hora antes del procedimiento. ${ }^{23-25}$

En este estudio se encontró, que los odontólogos generales usan, en baja proporción, antimicrobianos con fines profilácticos en pacientes con compromiso sistémico. Una posible explicación a estos resultados, es la falta de capacitación por parte de los odontólogos generales en temas relacionados con endodoncia, diagnóstico y terapéutica antimicrobiana; sin embargo, se percibió un mayor interés por la actualización en otras áreas de la odontología, una explicación similar fue sugerida por Zarik y Levin. ${ }^{2}$ Por otra parte, el poco tiempo de duración de la consulta odontológica no permite que el odontólogo general realice un diagnóstico adecuado, lo cual puede influir en la terapia farmacológica que se recomienda.

Otra posible interpretación de los hallazgos anteriores, se basa en el supuesto que los odontólogos generales deciden realizar el tratamiento endodóntico pero primero recurren al uso de antimicrobianos para resolver el problema infeccioso inicial y, posteriormente, llevar a cabo, la endodoncia. Es importante que cuando el odontólogo general realice un diagnóstico de una enfermedad pulpar o periapical de origen pulpar, se valore de forma integral la condición clínica y se considere la remisión al endodoncista, cuando el caso lo amerite.

Una fortaleza de este estudio, consiste en la formación académica del personal encargado de la aplicación del cuestionario durante las entrevistas personales y la recolección de la información, quienes eran odontólogos residentes del último año de la Especialización en Endodoncia, debidamente entrenados y capacitados para el diagnóstico y tratamiento relacionado con las patologías pulpares; este aspecto, permitió también resolver de manera adecuada las preguntas de los odontólogos generales encuestados y darles orientación sobre el manejo de las patologías pulpares y periapicales de origen pulpar.

Otro aspecto importante consiste en el mecanismo de aplicación de la encuesta la cual no fue autodiligenciada sino que se realizó mediante entrevista directa y administrada. De esta manera, se controló, en buena medida, la calidad de las respuestas obtenidas. También cabe destacar el alto nivel de interés por parte de los odontólogos encuestados, los cuales expresaron sus inquietudes y dudas, que fueron atendidas por los integrantes del equipo de investigación.

Finalmente, se puede concluir que una alta proporción de odontólogos generales usaron antimicrobianos sistémicos en patologías pulpares y periapicales de origen pulpar aún, cuando no estén indicados. El antimicrobiano más prescrito fue la amoxicilina. Una baja proporción de odontólogos usan antimicrobianos sistémicos con fines profilácticos. Es esencial, promover mediante actividades educativas dirigidas al odontólogo general, el uso correcto de antimicrobianos en cuanto a la indicación, elección, 
dosis, frecuencia de administración y duración de la terapia, así como recomendar la remisión al especialista en endodoncia, cuando el caso lo amerite. Así mismo, se requiere ampliar el tiempo de la consulta odontológica para poder realizar diagnósticos adecuados y tratamientos óptimos.

\section{BIBLIOGRAFÍA}

1. Walton RE, Torabinejad M. Endodoncia Principios y Práctica Clínica. 2da. Edición. Philadelphia: Interamericana McGraw-Hill; 1997.

2. Zadik Y, Levin L. Clinical decision making in restorative dentistry endodontics and antibiotic prescription. J Dent Educ [en línea] Jan 2008. [Fecha de acceso: marzo de 2008]; 72: 81 - 86. URL disponible en: http://www. jdentaled.org/cgi/content/full/72/1/81

3. Yingling NM, Byrne BE, Hartwell GR. Antibiotic use by members of the American Association of Endodontists in the year 2000: Report of a National Survey. J Endod 2002; 28: 396 - 404.

4. Rodríguez $\mathrm{H}$, Solar O. Uso indiscriminado de tetraciclina en afecciones bucales de origen odontológico. Rev Cub Estomatol [en línea]; 2007 [Fecha de acceso: marzo de 2008]; 44 (1). URL disponible en: http://scielo.sld.cu/scielo.php?

5. Ensinas P. Análisis de los hábitos de medicación antibiótica por parte de odontólogos generales ante diferentes patologías pulpares [en línea] 2007. [Fecha de acceso: marzo 30 de 2008]. URL disponible en: http://www.carlosboveda.com/englishsite/dentalguest.htm.

6. Tobón F. Estudio sobre automedicación en la Universidad de Antioquia. Latreia (Medellín). Diciembre 2002; 15: 242 - 247

7. Pérez H. Farmacología y Terapéutica Odontológica. 2 da. Ed. Bogotá: Celsus, 2005.

8. Caviedes J, Estévez MC, Rojas PA. Antibióticos en el manejo de las infecciones odontogénicas de origen endodóntico [en línea] 2006. [Fecha de acceso: abril de 2008]. Artículos de Revisión Posgrado de Endodoncia. URL disponible en: http://www.javeriana.edu.co/academiapgendodoncia/ i_a_revision $18 . h \mathrm{hml}$.

9. Cabrera $\mathrm{CE}$, Gómez RF, Zúñiga $\mathrm{AE}$. La resistencia de bacterias a antibióticos, antisépticos y desinfectantes una manifestación de los mecanismos de supervivencia y adaptación. Colombia Med 2007; 38: 149 - 158.

10. Ingle J. Endodoncia. 5ta. Ed. México: Mc Graw Hill, 2004.

11. Sigurdsson A. Pulpal diagnosis. Endod Topics 2003; 5: $12-25$.

12. Gatewood RS, Himel VT, Dorn SO. Treatment of the endodon- tic emergency: a decade later. J Endod 1990; 16: 284 - 291.

13. Whitten BH, Gardiner DL, Jeansonne BG, Lemon RR. Current trends in endodontics treatment-report of a national survey. J Am Dent Assoc 1996; 127: 1333 - 1341.

14. Milian VPM, Quiros EM, Boza MD, Santos PN, Martín AC. Caracterización del uso de antimicrobianos en las infecciones estomatológicas en un área de salud de Cuba. Int J Odontostomat 2007; 1: 177 - 184.

15. Abbot PV, Hume W, Pearman JW. Antibiotics and endodontics. Aust Dent J 1990; 35: 50 - 60.

16. Sutherland S. Antibiotics do not reduce toothache caused by irreversible pulpitis. Are systematic antibiotics effective in providing pain relief in people who have irreversible pulpitis? Evid Based Dent 2005; 6: 67.

17. Fica A. Manejo de la faringoamigdalitis estreptocócica en pacientes adultos o adolescentes. Rev Chil Infect 2002; 19: 79 - 91.

18. Fouad AF. Are antibiotics effective for endodontic pain? An evidence based review. Endod Topics 2002; 3: 52 - 66.

19. Fayad I. Presencia de microorganismos productores de Betalactamasa en dientes con periodontitis apical crónica supurativa. Revista Federación Odontológica Colombiana 2005; 25: 15 - 22.

20. Dumsha T, Gutmann J. Problems in Managing Endodontic Emergencies. En: Gutmann J, Dumsha T, Lovdahl P, Hovland E. Problem Solving in Endodontics: Prevention, Identification and Management. 2da. Ed. St. Louis: Mosby 1998. p. 174 - 189, p. 229 - 252.

21. Planells del Pozo P, Barra Soto MJ. Antibiotic prophylaxis in pediatric odontology. An update. Medicina Oral, Patología Oral y Cirugía Bucal 2006; 11: 352 - 357.

22. Retana AG. Protocolo de atención odontológica en pacientes con trastornos hematológicos. Revista Rhombus. 2007; 3.

23. Brincat M, Savarrio L, Saunders W. Endodontics and infective endocarditis - is antimicrobial chemoprophylaxis required? Int Endod J 2006; 39: 671 - 682.

24. Wilson W, Taubert KA, Gewitz M, Lockhart PB, Baddour LM, Levison M, et al. Prevention of infective endocarditis: Guidelines from the American Heart Association: a guideline from the American Heart Association Rheumatic Fever, Endocarditis and Kawasaki Disease Committee, Council on Cardiovascular Disease in the Young, and the Council on Clinical Cardiology, Council on Cardiovascular Surgery and Anesthesia, and the Quality of Care and Outcomes Research Interdisciplinary Working Group. J Am Dent Assoc 2007; 138: 739 - 745, 747 - 760.

25. Singh J. Antibiotic prophylaxis for endocarditis: time to reconsider. Aust Dent J Medications Supplement 2005; 50: 60 - 68 .

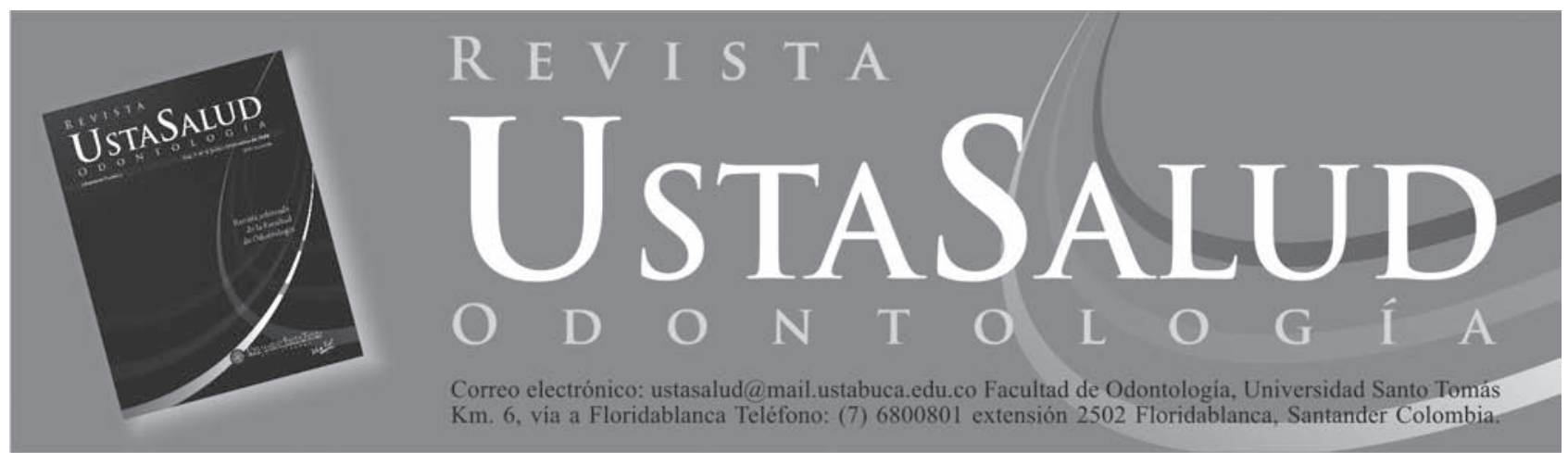

